\title{
Risk Factors associated with COVID-19 Infection among Contacts of Index Cases in Saudi Arabia: A Case-Control Study
}

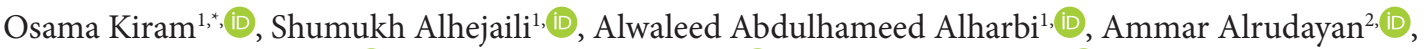

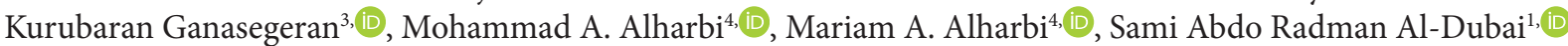 \\ ${ }^{1} J o i n t$ Program of Preventive Medicine Post Graduate Studies, Ministry of Health, Al-Madinah 41311, Saudi Arabia \\ ${ }^{2}$ Occupational Health Administration, Ministry of Health, Al-Madinah 42312, Saudi Arabia \\ ${ }^{3}$ Clinical Research Center, Seberang Jaya Hospital, Ministry of Health Malaysia, Penang 13700, Malaysia \\ ${ }^{4}$ Faculty of Medicine, King Abdulaziz University, Jeddah, Saudi Arabia
}

\section{ARTICLE INFO}

Article History

Received 19 July 2021

Accepted 13 November 2021

Keywords

COVID-19

infection

risk factors

Saudi Arabia

\begin{abstract}
This study was aimed to determine the risk factors associated with COVID-19 infection among contacts of index cases in Saudi Arabia. This unmatched case-control study was conducted among contacts of confirmed COVID-19 cases in April and May 2020 in Al-Madinah, Saudi Arabia. A total of 118 cases and 115 controls were included in this study. All cases and controls were interviewed via telephone by using a structured questionnaire that included two sections. The first section included participants' personal and demographic information, and the second part included questions on the degree of distancing and the duration of contact prior to the appearance of the first symptom, seasonal influenza vaccination, and the nature of the relationship with the index case patient. The majority of cases (83.1\%) and controls $(67.2 \%)$ were males. The mean standard deviation age for the whole sample was 35.3 (10.6) years, and their ages ranged from 18 to 63 years. In the multivariate analysis, the risk for COVID-19 infection was increased by age [Adjusted Odds Ratio $(\mathrm{aOR})=2.2 ; 95 \%$ Confidence Interval $(\mathrm{CI}), 1.1-4.8, p=0.046$ ], and was higher among family members $(\mathrm{aOR}=10 ; 95 \% \mathrm{CI}, 3.3-20.0 ; p=0.001)$, coworkers $(\mathrm{aOR}=20.0 ; 95 \% \mathrm{CI}, 4.4-50.0 ; p<0.001)$, and those who communicated with an index case at a distance of $<1.5 \mathrm{~m}(\mathrm{aOR}=4.3 ; 95 \% \mathrm{CI}, 1.9-9.8 ; p=0.001)$.
\end{abstract}

() 2021 Dr. Sulaiman Al Habib Medical Group. Publishing services by Atlantis Press International B.V. This is an open access article distributed under the CC BY-NC 4.0 license (http://creativecommons.org/licenses/by-nc/4.0/).

\section{INTRODUCTION}

COVID-19 is an infectious disease caused by SARS-CoV-2. The disease has caused a global pandemic, affecting people in more than 200 countries to date [1]. As of November 11, 2020, approximately $51,251,715$ people have tested positive for the disease, and $1,270,930(2.48 \%)$ of these cases had died [1]. In Saudi Arabia, approximately 351,455 positive cases were detected as of November 11,2020 , with a mortality rate of $1.59 \%$ [1].

Case definitions and clinical evaluations are crucial to confirm if an individual's reported symptoms and signs are compatible with COVID-19 infection, and to subsequently decide if the individual should be isolated or tested [2]. The incubation period ranges from 5 to 14 days, with a variety of reported symptoms such as fever, cough, fatigue, muscle aches, headache, loss of taste or smell, sore throat, conjunctivitis, rash, diarrhea, or more severe manifestations such as shortness of breath, chest pain, and loss of speech or movement [3].

"Corresponding author. Email: Kiramosama@gmail.com

Peer review under responsibility of the Dr. Sulaiman Al Habib Medical Group Data availability statement: The data that support the findings of this study are available from the corresponding author $[\mathrm{OK}]$ upon reasonable request.
The transmission dynamics for this novel pathogen differs across countries and populations. Although the majority of the cases were community-acquired transmissions, there were countries that reported clustered and sporadic spread as well [4]. To date, scientists and public health experts have no solid evidence to confirm the exact etiology and mechanism of disease transmission; however, epidemiological trends of positive cases have provided some clues on possible risk factors for COVID-19 infection. As people's susceptibility to COVID-19 differ across settings or conditions, it is crucial to identify vulnerable individuals who require quarantine or targeted testing to contain the outbreak. Various reports in the literature have noted clustering of household members who were in contact with COVID-19 patients [5-7], and those who lived or communicated with confirmed COVID-19 patients were observed to be highly prone to infection [8]. These transmissions were likely to occur through cough, sneeze, or direct contact with the virus on contaminated surfaces [9]. In asymptomatic infected individuals, the absence of symptoms may lead them not to be screened or remain undetected. Such conditions may predispose asymptomatic close contacts to become an important source of explosive virus transmissions, which substantially warrants strict monitoring of close contacts via early screening and detection [10]. Older people and people with comorbidities such as diabetes, obesity, or hypertension had greater risk for COVID-19 infection [11]. Having close 
contacts with infected people, living circumstances in confined spaces, and nonadherence to protective behaviors such as use of face masks, hand hygiene, physical distancing, and type of contacts with index cases have been postulated to escalate the risk of infection $[2,12,13]$. This study was aimed to determine the risk factors associated with COVID-19 infection among contacts of index cases in Saudi Arabia.

\section{MATERIALS AND METHODS}

\subsection{Study Design, Setting, and Participants}

This unmatched case-control study was conducted among contacts of confirmed COVID-19 cases during April and May 2020 in Al-Madinah, Saudi Arabia. The study recruited 124 primary cases of COVID-19 as the source of infection (index cases). The contacts of those patients were followed up. An infected contact was recruited as a case. For each case, a control was selected from the panel of contacts within the same primary case after being tested negative for the second time within 14 days, after the first test result. As the COVID-19 incubation period was thought to extend up to 14 days [14-16], the current study followed the WHO Clinical Interim Guidelines that assured post-14 days as free of COVID-19 infectiousness [17]. This ensured that the recruited controls were free from the disease.

The case and control group information was obtained from the Public Health Administration of Saudi Arabia together with a list of telephone contact numbers. COVID-19 nasal swab results [Polymerase Chain Reaction (PCR) nasopharyngeal test] and phone numbers were continuously updated to fit the study's sample size. The Public Health Administration was contacted daily for new cases and to check if any of the participants have changed their phone numbers. This enabled the study investigators to update the list of contact numbers of the participants recruited in this study. This ensured that no contacts were lost. If the contact number of the participants were lost and if the study investigators could not follow up the participants, those individuals would be excluded from the study. A total of 118 cases and 115 controls were included in this study.

\subsection{Operational Definitions}

Contacts were defined as any adult individuals who have a close relationship (within $6 \mathrm{ft}$ of an infected person for a total of 15 min or more) with a laboratory-confirmed COVID-19 patient [18]. All participants were subjected to two PCR tests, so if any of the contacts tested positive and diagnosed with COVID-19 in the second test, they would be moved from the control group to the case group. Subsequently, all new cases (who were prior contacts) were called to obtain a full medical history and epidemiological links information, including contact history, even if they were in isolation.

Cases were defined as (inclusion criteria) all adult patients who tested positive after the second test for COVID-19 in the months of April and May 2020. All patients were followed up by the Public Health Administration of Saudi Arabia regardless of the testing center in which they were tested-governmental or private hospitals, primary health centers, or through community surveillance programs.

All cases must have gone through one maximum incubation period (14 days) prior to being included in the study to ensure that no symptoms were missed. Controls were defined as any adult contacts of a confirmed COVID-19 case who tested negative twice, after being in contact with the index case.

Symptomatic cases include individuals who complained of at least one classical COVID-19 symptoms according to the Center for Disease Control case definition (fever, chills, cough, shortness of breath or difficulty breathing, fatigue, muscle or body aches, headache, loss of taste or smell, sore throat, congestion or runny nose, nausea or vomiting, diarrhea), after exposure to the virus [19]. Asymptomatic cases referred to infected individuals who showed none of these symptoms, which may have led them not to be screened or remained undetected.

The first and second test results referred to participants in the current study who underwent two PCR tests; and if the second test result returned negative, then the individual (case) would be moved to the control (contact) group [19]. The exclusion criteria for the cases were children. The exclusion criteria for controls were children and any COVID-19 positive individuals at 14 days after contact with a case. Both cases and controls were excluded if they refused participation or have not responded to telephone calls for two successive days.

\subsection{Data Collection}

Data were collected by trained doctors through telephone calls. A predesigned data collection sheet was used. The data collection sheet consisted of two sections; the first part included participants' personal and demographic information (age, job, sex, marital status, nationality, smoking status, education, monthly income, blood group, height, weight, and residence information), whereas the second part included questions on the degree of distancing and the duration of contact prior to the appearance of the first symptom, seasonal influenza vaccination, and the nature of the relationship with the index case. In addition, cases were asked about the symptoms of the disease.

\subsection{Statistical Analysis}

Data were entered and managed using SPSS version 25 (IBM Corp., Armonk, NY, USA). Frequency and percentages were reported for all categorical variables, whereas mean and Standard Deviations (SD) were reported for continuous variables. Chi-square test and simple binary logistic regressions were performed to determine the association between categorical variables in the univariate analysis. Crude Odds Ratios (cOR) were obtained. Multiple logistic regression analysis was applied to determine the most important factors to be associated with COVID-19 infection. Adjusted Odds Ratios (aOR) were obtained. Hosmer-Lemeshow goodness-of-fit test and the Nagelkerke $R^{2}$ were obtained in the multiple logistic regression analysis to determine a parsimonious model. The accepted level of significance was set at $p<0.05$. 


\section{RESULTS}

\subsection{Sample Characteristics}

The majority of cases $(83.1 \%)$ and controls (67.2\%) were males. The mean (SD) age for the whole sample was 35.3 (10.6) years, and their ages ranged from 18 to 63 years. The mean (SD) age for the cases was 37.6 (10.8) years, whereas that for controls was 33.1 (9.7) years. Regarding the clinical characteristics of cases, the first test result was positive in $91.5 \%$ of the cases, whereas the second test results was positive for all. Most cases were symptomatic $(73.7 \%)$ (Table 1). The majority of the cases reported to have fever (44.6\%), change in sense of smell or taste $(32.2 \%)$, or being fatigued $(30.5 \%)$ (Figure 1).

\subsection{Risk Factors associated with COVID-19 Infection among Contacts}

The risk of infection with COVID-19 was higher in males [COR $=2.4 ; 95 \%$ Confidence Interval (CI), 1.3-4.4], among those aged $>35$ years $(\mathrm{cOR}=2.0 ; 95 \% \mathrm{CI}, 1.2-3.4)$, married $(\mathrm{cOR}=2.2 ; 95 \%$ CI, 1.2-3.9), non-Saudis ( $\mathrm{COR}=2.3$; 95\% CI, 1.4-4.1), and private employees $(\mathrm{cOR}=2.6 ; 95 \% \mathrm{CI}, 1.4-4.9)$. The risk was also higher among those who did not adhere to protective behaviors during contact with an index case $(\mathrm{cOR}=1.7 ; 95 \% \mathrm{CI}, 1.3-2.9)$ and among those who communicated with an index case at a distance of $<1.5 \mathrm{~m}(\mathrm{cOR}=2.7 ; 95 \% \mathrm{CI}, 1.5-4.9)$. As compared with being

Table 1 Characteristics of cases and COVID-19 detection $(n=118)$

\begin{tabular}{lcc}
\hline Characteristics & Frequency $(\boldsymbol{n})$ & Percentage (\%) \\
\hline First test result & & \\
$\quad$ Positive & 108 & 91.5 \\
Negative & 10 & 8.5 \\
Second test result & & \\
$\quad$ Positive & 118 & 100 \\
Negative & 0 & 0 \\
Days to symptoms onset since contact with index case \\
$\leq 5$ & 37 & 31.4 \\
$>5$ & 81 & 68.6 \\
Infection spectrum & & \\
Asymptomatic & 31 & 26.3 \\
Symptomatic & 87 & 73.7 \\
\hline
\end{tabular}

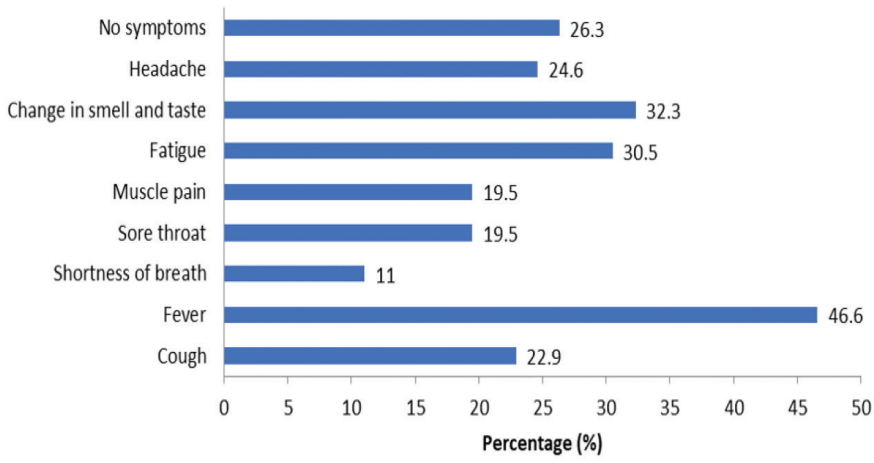

Figure 1 Clinical manifestations of COVID-19. in contact with unknown people, the risk of infection was higher among coworkers (cOR $=10.7 ; 95 \% \mathrm{CI}, 3.3-35.1)$, family members $(\mathrm{cOR}=6.0 ; 95 \% \mathrm{CI}, 2.9-12.3)$, and healthcare providers $(\mathrm{cOR}=$ 5.0; 95\% CI, 1.1-10.0). These associations were statistically significant (Table 2). There was no association between individual's blood group and risk of COVID-19 infection.

\subsection{Risk Factors associated with COVID-19 Infection by Multiple Logistic Regression}

Risk factors that were significantly associated with positive testing of COVID-19 infection in the univariate analysis were included in the multiple logistic regression analysis. The final multivariable logistic regression model found that the risk for COVID-19 infection increased with age $(\mathrm{aOR}=2.2 ; 95 \% \mathrm{CI}, 1.1-4.8)$, and was higher among family members $(\mathrm{aOR}=10$; 95\% CI, 3.3-20.0), coworkers ( $\mathrm{aOR}=20.0 ; 95 \% \mathrm{CI}, 4.4-50.0)$, and those who communicated with an index case at $<1.5 \mathrm{~m}(\mathrm{aOR}=4.3$; 95\% CI, 1.9-9.8). The total model was significant $(p<0.001)$ and accounted for $39 \%$ of the variance (Table 3).

\section{DISCUSSION}

The aim of this study was to determine the risk factors associated with positive testing of COVID-19 infection among contacts of index cases in Al-Madinah region, Saudi Arabia. The final regression model concluded that older adults ( $>35$ years old), family members or coworkers of an index case, and those had who encounters at a shorter contact distance $(<1.5 \mathrm{~m})$ with an index case had higher risk to test positive for COVID-19.

This study found that coworkers had the highest risk to test positive for COVID-19, followed by family members of an index case. Although the association between the contact's relationship with an index case and infection risks was statistically significant in the present study, and consistent with previous studies, their effect sizes between groups varied across different populations. Studies from China [6,20] and Thailand [21] found that family members had greater risk of infections as compared with other susceptible individuals. Two plausible explanations for such differing associations could be postulated: (1) variation in the definition of contact types used in different studies, which could have influenced the size of a household-such as family members consisting of a spouse only, or including relatives or other members living in the household with an index case, thereby posing greater risk of transmissions [22,23]; (2) countries that implemented stringent measures such as lockdowns and work-from-home policies may have escalated exposure of index cases to their family members while at home. In contrast, plausible explanation of coworkers having greater risk of infection, as found in this study, could be attributed to the nature of employment among contacts in Saudi Arabia, either being in government or private employment. Government employees in this country can afford to stay out of their employment settings because they are on government payroll, and as such, they can go on paid leave or adopt work-from-home policy, whereas private employees are hardly provided with paid leaves, and are thus forced to go to work, thereby risking their coworkers' health. This explanation could be 
Table 2 Risk factors associated with COVID-19 infection among contacts of index cases

\begin{tabular}{|c|c|c|c|c|}
\hline \multirow{2}{*}{ Risk factors } & \multirow{2}{*}{$\begin{array}{c}\text { Cases } \\
n(\%)\end{array}$} & \multirow{2}{*}{$\begin{array}{c}\text { Control } \\
n(\%)\end{array}$} & \multirow{2}{*}{ cOR } & \multirow{2}{*}{$95 \% \mathrm{CI}$} \\
\hline & & & & \\
\hline \multicolumn{5}{|l|}{ Sex } \\
\hline Male & $98(83.1)$ & $80(67.2)$ & $2.4^{*}$ & $1.3-4.4$ \\
\hline Female & $20(16.9)$ & $39(32.8)$ & 1 & - \\
\hline \multicolumn{5}{|l|}{ Age (years) } \\
\hline$\leq 35$ & $55(46.6)$ & $76(63.9)$ & 1 & - \\
\hline$>35$ & $63(53.4)$ & $43(36.1)$ & $2.0^{*}$ & $1.2-3.4$ \\
\hline \multicolumn{5}{|l|}{ Marital status } \\
\hline Married & $95(80.5)$ & $78(65.5)$ & $2.2^{*}$ & $1.2-3.9$ \\
\hline Single & $23(19.5)$ & $41(34.5)$ & 1 & - \\
\hline \multicolumn{5}{|l|}{ Nationality } \\
\hline Saudi & $32(27.1)$ & $56(47.1)$ & 1 & - \\
\hline Non-Saudi & $86(72.9)$ & $63(52.9)$ & $2.3^{*}$ & $1.4-4.1$ \\
\hline \multicolumn{5}{|l|}{ Employment } \\
\hline Unemployed & $21(17.8)$ & $39(34.2)$ & 1 & - \\
\hline Government & $23(19.5)$ & $22(19.3)$ & 1.9 & $0.9-4.3$ \\
\hline Private & $74(62.7)$ & $53(46.5)$ & $2.6^{*}$ & $1.4-4.9$ \\
\hline \multicolumn{5}{|l|}{ Income level (SAR) } \\
\hline$<5000$ & $78(66.1)$ & $82(68.9)$ & 1.1 & $0.7-1.4$ \\
\hline$\geq 5000$ & $40(33.9)$ & $37(31.1)$ & 1 & - \\
\hline \multicolumn{5}{|l|}{ Education } \\
\hline School & $54(45.8)$ & $66(55.5)$ & 1 & - \\
\hline University & $64(54.2)$ & $53(44.5)$ & 1.4 & $0.9-2.5$ \\
\hline \multicolumn{5}{|l|}{ Residence } \\
\hline Apartment/villa & $92(78.6)$ & $103(86.6)$ & 1.6 & $0.9-3.3$ \\
\hline Dorms/workers residence & $25(21.4)$ & $16(13.4)$ & 1 & - \\
\hline \multicolumn{5}{|l|}{ BMI } \\
\hline Normal & $35(29.7)$ & $46(38.7)$ & 1 & - \\
\hline Overweight/obese & $83(70.3)$ & $73(61.3)$ & 1.4 & $0.4-1.1$ \\
\hline \multicolumn{5}{|l|}{ Smoking } \\
\hline Yes & $10(8.5)$ & $17(14.3)$ & 0.6 & $0.2-1.3$ \\
\hline No & $108(91.5)$ & $102(85.7)$ & 1 & - \\
\hline \multicolumn{5}{|l|}{ Shisha } \\
\hline Yes & $13(11.0)$ & $17(14.3)$ & 0.7 & $0.3-1.6$ \\
\hline No & $105(89.0)$ & $102(85.7)$ & 1 & - \\
\hline \multicolumn{5}{|l|}{ Chronic diseases $^{\mathrm{a}}$} \\
\hline Yes & $28(23.7)$ & $18(15.1)$ & 0.6 & $0.3-1.1$ \\
\hline No & $90(76.3)$ & $101(84.9)$ & 1 & - \\
\hline \multicolumn{5}{|c|}{ Protective behaviors during contact with index case $\mathrm{b}^{\mathrm{b}}$} \\
\hline Yes & $56(47.5)$ & $41(34.5)$ & 1 & - \\
\hline No & $62(52.5)$ & $78(65.5)$ & $1.7^{*}$ & $1.3-2.9$ \\
\hline \multicolumn{5}{|l|}{ Relationship with index case } \\
\hline Family & $21(17.8)$ & $62(52.1)$ & $6.0^{*}$ & $2.9-12.3$ \\
\hline Coworkers & $28(23.7)$ & $12(10.1)$ & $10.7^{*}$ & $3.3-35.1$ \\
\hline Relatives/neighbors & $4(3.4)$ & $21(17.6)$ & 1.1 & $0.5-2.5$ \\
\hline Healthcare provider & $20(16.9)$ & $2(1.7)$ & $5^{*}$ & $1.1-10.0$ \\
\hline Unknown & $45(38.1)$ & $22(18.5)$ & 1 & - \\
\hline Contact distance with index c & case $(\mathrm{m})$ & & & \\
\hline$<1.5$ & $56(67.5)$ & $40(43.5)$ & $2.7^{*}$ & $1.5-4.9$ \\
\hline$\geq 1.5$ & $27(32.5)$ & $52(56.5)$ & 1 & - \\
\hline Blood group $\mathrm{ABO}(n=186)$ & & & & \\
\hline $\mathrm{O}$ & $45(44.1)$ & $39(46.4)$ & 1 & - \\
\hline $\mathrm{A}$ & $39(38.2)$ & $29(34.5)$ & 1.2 & $0.6-2.2$ \\
\hline B & $13(12.7)$ & $14(16.7)$ & 0.8 & $0.3-1.9$ \\
\hline $\mathrm{AB}$ & $5(4.9)$ & $2(2.4)$ & 2.2 & $0.4-11.8$ \\
\hline
\end{tabular}

${ }^{a}$ Contacts have at least one of the following diseases: diabetes, hypertension, asthma, or ischemic heart disease as documented in medical history records. ${ }^{\mathrm{b}}$ Contacts selfreported practice of protective measures—hand sanitizing and masking. "Statistically significant at $p<0.05$. BMI, body mass index; SAR, Saudi Riyal.
Table 3 Risk factors associated with COVID-19 infection by multivariate logistic regression analysis (backward elimination)

\begin{tabular}{|c|c|c|c|c|}
\hline \multirow{2}{*}{ Risk factors } & \multirow{2}{*}{$B$} & \multirow{2}{*}{$\operatorname{Exp}(B)^{\mathrm{a}}$} & \multicolumn{2}{|c|}{ 95\% CI } \\
\hline & & & Lower & Upper \\
\hline \multicolumn{5}{|l|}{ Age (years) } \\
\hline$\leq 35$ & Ref & Ref & Ref & Ref \\
\hline$>35$ & 0.8 & $2.2^{*}$ & 1.1 & 4.8 \\
\hline \multicolumn{5}{|c|}{ Relationship with index case } \\
\hline Family & -2.5 & $10.0^{*}$ & 3.3 & 20.0 \\
\hline Coworkers & -3.1 & $20.0^{*}$ & 4.4 & 50.0 \\
\hline Relatives/neighbors & -0.5 & 1.3 & 0.4 & 6.6 \\
\hline Healthcare provider & 0.6 & 1.9 & 0.3 & 11.3 \\
\hline Unknown & Ref & Ref & Ref & Ref \\
\hline \multicolumn{5}{|c|}{ Contact distance with index case (m) } \\
\hline$<1.5$ & 1.4 & $4.3^{*}$ & 1.9 & 9.8 \\
\hline$\geq 1.5$ & Ref & Ref & Ref & Ref \\
\hline
\end{tabular}

${ }^{\mathrm{a}} \operatorname{Exp}(B)$ gives the adjusted odds ratio (aOR). "Statistically significant at $p<0.05$.

Ref, reference category.

supported with results obtained at the univariate level (although this was eliminated at the multivariate analysis), whereby a statistically significant association was found between employment and risk of COVID-19 infection, as it was found that private employees were more likely to be at risk of infection as compared with government employees.

Advancing age was postulated to increase COVID-19 infection risks. Based on the age-specific transmissibility epidemiological model, it was found that COVID-19 showed high transmissibility rates in those aged 45-64 years old and in elderly individuals, aged 65 years or older [24]. This study found that contacts older than 35 years had twice the odds to test positive for COVID-19 as compared with younger groups. Similar consistencies were observed in previous studies from China [20] and Mexico [25], but inconsistent with a study conducted in Singapore [13].

SARS-CoV-2, which is the causative pathogen for the transmission of COVID-19, mediates respiratory infection via droplets generated while talking, breathing, coughing, or sneezing. These droplets, which are circular in nature with a diameter of approximately $5 \mu \mathrm{m}$ rapidly, fall to the ground under gravity, which limits transmission of $<1 \mathrm{~m}$ (distance) under normal breathing [26], or more than $1 \mathrm{~m}$ under forceful sneezing or coughing [27]. Most countries have advocated physical distancing from 1 to $2 \mathrm{~m}$ to avoid transmission of SARS-CoV-2. This study found that those in contact with an index case at $<1.5 \mathrm{~m}$ were more likely to be at risk to test positive for COVID-19. Similar consistencies were observed among samples from different situations, such as transmissions among train passengers who were seated nearby [28], and among healthcare workers who generally consume food in confined spaces [29].

At the univariate level, it was found than males were more likely to be at risk to test positive for COVID-19, and this association was statistically significant. However, the bulk of previous studies found otherwise $[2,10,12,13,29]$. The finding that males were more likely to be infected with COVID-19 in this study should be interpreted with caution, as the statistically significant association between sex and infection risk might be attributable to the relatively high number of male individuals recruited in this sample. Married contacts were more likely to test positive in the current study, because spouses of index cases who live in the same household would have 
been greatly exposed under confined spaces during interactions, yet the risk of infection may be greater if their partner happens to be asymptomatic.

This study found that non-Saudis were more likely to test positive for COVID-19 as compared with Saudi nationals, and this association was statistically significant. Migrants, either legal or illegal, may not adhere to standard operating procedures as determined by the country owing to lack of awareness, language barriers, lack of entitlement to country's healthcare provision, or unwillingness/ inability to seek healthcare services owing to fear of deportation. In addition, migrants' living circumstances under permissive conditions, crowded situations, or in confined spaces may increase the risk of transmission among local contacts [30].

As the exact cause of infection could not be determined to break the chain of infection for this relatively novel virus, nonpharmacological interventions such as use of face masks and hand sanitizing were crucial to control the "aerosol" transmission and contamination of the virus. This study found that those who did not adhere to protective behaviors during contact with an index case were more likely to test positive for COVID-19. Similar findings were observed in previous studies [12,29].

Although emerging literature has reported $\mathrm{ABO}$ blood groups as a risk factor for COVID-19 infection [31,32], this postulation seems premature and controversial with limited evidence of robust study designs. A recent meta-analysis reported that those with blood group A were associated with a higher risk of contracting COVID-19 whereas people with blood group O seem to be at a lower risk [33]. The present study, however, failed to determine any associations between an individual's blood group and risk of COVID-19 infection.

Among symptomatic cases, the most commonly reported symptoms in this study were fever, change in sense of smell or taste, and fatigue. There were differing symptoms reported across different studies and populations. A study from China found that cough and fever were the most common symptoms reported [12], whereas another study from Saudi Arabia found cough, fever, and sore throat to be commonly encountered [2].

\subsection{Study Limitations}

The limitations for a case-control design used in this study should be acknowledged. Because of the retrospective nature of this study, the contacts of index cases may have been prone to recall bias, as some contacts may have found it somewhat difficult to recall certain details of the multiple risk factors assessed in this study. One such example is when they were asked to recall the distance between contacts and index cases during communication. In addition, the relatively small sample size may limit generalizability of the study findings. The findings of this study should be interpreted with caution as the interpretation of "risk factors" was not powered to establish causality for apprehension as a diagnostic, prognostic, treatment effect, or the establishment as an etiological factor [34]. Instead, in such observational studies that yield regression models, the attributes or exposures examined are primarily hypothesis generating instead of hypothesis testing; thus, the interpretation should be conceptualized as "associated factors," that is, how much likely would this factor be associated with the outcome measure $[35,36]$.

\section{CONCLUSION}

Most cases in this study manifested symptomatic COVID-19 infection. Older adults, family members, or coworkers of an index case, and those who had encounters at shorter contact distances with an index case had higher risk to test positive for COVID-19. It is recommended that rigorous contact tracing initiatives to detect asymptomatic carriers and practice of physical distancing measures be adopted to facilitate a conducive living environment, which is crucial to break the chain of transmission.

\section{CONFLICTS OF INTEREST}

The authors declare they have no conflicts of interest.

\section{AUTHORS' CONTRIBUTION}

OK and SARA contributed in study conceptualization and writing (review and editing) the manuscript. SA, AA, MoAA, MaAA and $K G$ contributed in data curation, formal analysis, and writing (original draft). AAA contributed in acquisition and project administration. SARA supervised the project, whereas $\mathrm{OK}$ and $\mathrm{KG}$ were involved in formal analysis and writing (original draft) the manuscript. All authors read and approved the final version of the manuscript.

\section{ETHICAL APPROVAL}

The study was conducted according to the guidelines of the Declaration of Helsinki, and approved by the Ethics Committee of the Directorate of Health in Al-Madinah (No: H-03-M-084).

\section{INFORMED CONSENT STATEMENT}

Informed consent was obtained from all participants involved in the study. In view of movement restrictions during pandemic times, oral consent was obtained through the telephone from all participants. The data collector would read the consent form to the participant, and then answer any questions that the participant may have. Eventually, when the data collector felt confident that the participant has understood the information, the data collector would document the consent by participant number, time, and date. Confidentiality was assured and objectives of the study were explained to the participants.

\section{REFERENCES}

[1] WHO Coronavirus Disease (COVID-19) Dashboard. Available from: https://covid19.who.int/table (accessed December 22, 2020).

[2] Alsofayan YM, Althunayyan SM, Khan AA, Hakawi AM, Assiri AM. Clinical characteristics of COVID-19 in Saudi Arabia: a national retrospective study. J Inf Public Health 2020;13;920-5.

[3] WHO Health Topics. Available from: https://www.who.int/ health-topics/coronavirus\#tab=tab_3 (accessed December 22, 2020). 
[4] Fang Y, Nie Y, Penny M. Transmission dynamics of the COVID-19 outbreak and effectiveness of government interventions: a datadriven analysis. J Med Virol 2020;92;645-59.

[5] Song R, Han B, Song M, Wang L, Conlon CP, Dong T, et al. Clinical and epidemiological features of COVID-19 family clusters in Beijing, China. J Infect 2020;81;e26-e30.

[6] Xia Xy, Wu J, Liu Hl, Xia H, Jia B, Huang Wx. Epidemiological and initial clinical characteristics of patients with family aggregation of COVID-19. J Clin Virol 2020;127;104360.

[7] Qian G, Yang N, Ma AHY, Wang L, Li G, Chen X, et al. COVID-19 transmission within a family cluster by presymptomatic carriers in China. Clin Infect Dis 2020;71;861-2.

[8] Li W, Zhang B, Lu J, Liu S, Chang Z, Peng C, et al. Characteristics of household transmission of COVID-19. Clin Infect Dis 2020;71;1943-6.

[9] Hu P, Ma M, Jing Q, Ma Y, Gan L, Chen Y, et al. Retrospective study identifies infection related risk factors in close contacts during COVID-19 epidemic. Int J Infect Dis 2021;103; 395-401.

[10] Martinez-Fierro ML, Ríos-Jasso J, Garza-Veloz I, Reyes-Veyna L, Cerda-Luna RM, Duque-Jara I, et al. The role of close contacts of COVID-19 patients in the SARS-CoV-2 transmission: an emphasis on the percentage of nonevaluated positivity in Mexico. Am J Infect Control 2021;49;15-20.

[11] Sanyaolu A, Okorie C, Marinkovic A, Patidar R, Younis K, Desai P, et al. Comorbidity and its impact on patients with COVID-19. SN Compr Clin Med 2020;2;1069-76.

[12] Wu Y, Song S, Kao Q, Kong Q, Sun Z, Wang B. Risk of SARSCoV-2 infection among contacts of individuals with COVID-19 in Hangzhou, China. Public Health 2020;185;57-9.

[13] Ng OT, Marimuthu K, Koh V, Pang J, Linn KZ, Sun J, et al. SARSCoV-2 seroprevalence and transmission risk factors among high-risk close contacts: a retrospective cohort study. Lancet Infect Dis 2021;21;333-43.

[14] Guan Wj, Ni Zy, Hu Y, Liang Wh, Ou Cq, He Jx, et al. Clinical characteristics of coronavirus disease 2019 in China. N Engl J Med 2020;382;1708-20.

[15] Li Q, Guan X, Wu P, Wang X, Zhou L, Tong Y, et al. Early transmission dynamics in Wuhan, China, of novel coronavirusinfected pneumonia. N Engl J Med 2020;382;1199-207.

[16] Lauer SA, Grantz KH, Bi Q, Jones FK, Zheng Q, Meredith HR, et al. The incubation period of coronavirus disease 2019 (COVID-19) from publicly reported confirmed cases: estimation and application. Ann Intern Med 2020;172;577-82.

[17] World Health Organization (WHO). Criteria for releasing COVID-19 patients from isolation. Available from: https://www. who.int/news-room/commentaries/detail/criteria-for-releasingcovid-19-patients-from-isolation (accessed August 30, 2021).

[18] Centre for Disease Control. Contact tracing for COVID-19. Available from: https://www.cdc.gov/coronavirus/2019-ncov/ $\mathrm{php} /$ contact-tracing/contact-tracing-plan/contact-tracing.html (accessed August 30, 2021).

[19] Center for Disease Control. Symptoms of COVID-19. Available from: https://www.cdc.gov/coronavirus/2019-ncov/symptomstesting/symptoms.html (accessed August 30, 2021).

[20] Liu T, Liang W, Zhong H, He J, Chen Z, He G, et al. Risk factors associated with COVID-19 infection: a retrospective cohort study based on contacts tracing. Emerg Microbes Infect 2020;9; 1546-53.
[21] Phucharoen C, Sangkaew N, Stosic K. The characteristics of COVID-19 transmission from case to high-risk contact, a statistical analysis from contact tracing data. EClinicalMedicine 2020;27;100543.

[22] Koh WC, Naing L, Chaw L, Rosledzana MA, Alikhan MF, Jamaludin SA, et al. What do we know about SARS-CoV-2 transmission? A systematic review and meta-analysis of the secondary attack rate and associated risk factors. PLoS One 2020;15;e0240205.

[23] Xin H, Jiang F, Xue A, Liang J, Zhang J, Yang F, et al. Risk factors associated with occurrence of COVID-19 among household persons exposed to patients with confirmed COVID-19 in Qingdao Municipal, China. Transbound Emerg Dis 2021;68;782-8.

[24] Zhao ZY, Zhu YZ, Xu JW, Hu SX, Hu QQ, Lei Z, et al. A fivecompartment model of age-specific transmissibility of SARSCoV-2. Infect Dis Poverty 2020;9;117.

[25] Berumen J, Schmulson M, Alegre-Díaz J, Guerrero G, LarrivaSahd J, Olaiz G, et al. Risk of infection and hospitalization by Covid-19 in Mexico: a case-control study. medRxiv 2020 [Preprint].

[26] Lippi G, Henry BM, Bovo C, Sanchis-Gomar F. Health risks and potential remedies during prolonged lockdowns for coronavirus disease 2019 (COVID-19). Diagnosis (Berl) 2020;7; $85-90$.

[27] Jones NR, Qureshi ZU, Temple RJ, Larwood JPJ, Greenhalgh T, Bourouiba L. Two metres or one: what is the evidence for physical distancing in covid-19?. BMJ 2020;370;m3223.

[28] Hu M, Lin H, Wang J, Xu C, Tatem AJ, Meng B, et al. Risk of coronavirus disease 2019 transmission in train passengers: an epidemiological and modeling study. Clin Infect Dis 2021;72; 604-10.

[29] Çelebi G, Pişkin N, Çelik Bekleviç A, Altunay Y, Salc1 Keleş A, Tuz MA, et al. Specific risk factors for SARS-CoV-2 transmission among health care workers in a university hospital. Am J Infect Control 2020;48;1225-30.

[30] Guadagno, L. Migrants and the COVID-19 pandemic: an initial analysis. Migration Research Series $N^{\circ}$ 60. Geneva, Switzerland: International Organization for Migration (IOM); 2020. Available from: https://publications.iom.int/system/files/pdf/mrs-60.pdf (accessed December 22, 2020).

[31] Latz CA, DeCarlo C, Boitano L, Maximilian Png CY, Patell R, Conrad MF, et al. Blood type and outcomes in patients with COVID-19. Ann Hematol 2020;99;2113-18.

[32] Ray JG, Schull MJ, Vermeulen MJ, Park AL. Association between $\mathrm{ABO}$ and Rh blood groups and SARS-CoV-2 infection or severe COVID-19 illness: a population-based cohort study. Ann Intern Med 2021;174;308-15.

[33] Pourali F, Afshari M, Alizadeh-Navaei R, Javidnia J, Moosazadeh M, Hessami A. Relationship between blood group and risk of infection and death in COVID-19: a live meta-analysis. New Microbes New Infect 2020;37;100743.

[34] Huitfeldt A. Is caviar a risk factor for being a millionaire?. BMJ 2016;355;i6536.

[35] Tennant PWG, Murray EJ. The quest for timely insights into COVID-19 should not come at the cost of scientific rigor. Epidemiology 2021;32;e2.

[36] Westreich D, Edwards JK, van Smeden M. Comment on Williamson et al. (OpenSAFELY): the table 2 fallacy in a study of COVID-19 mortality risk factors. Epidemiology 2021;32;e1-e2. 